\title{
Direct patterning of nanometer-scale structures on insulating substrates with variable pressure electron beam lithography (VP-eBL)
}

\author{
Benjamin D. Myers* and Vinayak P. Dravid* \\ *NUANCE Center and Department of Materials Science and Engineering, Northwestern University, \\ 2220 Campus Drive, Evanston, IL 60208
}

Electron beam lithography $(e \mathrm{BL})$ is a common technique for the fabrication of nanometer-scale structures. The fabrication of 2-D structures as small as $10 \mathrm{~nm}$ can be achieved through exposure of an electron sensitive resist layer with a high energy $(30-100 \mathrm{keV})$ electron beam. Due to the high beam energy, a large portion of the incident electrons penetrates the resist layer and is deposited in the substrate. Given an electrically grounded substrate with sufficient conductivity, the excess charge is readily dissipated. However, in the case of an electrically insulating substrate, the charge can be trapped near the surface. This excess charge results in the generation of local electric fields of sufficient strength to deflect the incident beam and cause pattern distortion as shown in Figure 1a. Several methods have been employed to mitigate this issue including the use of a grounded metal overlayer (typically $\mathrm{Al}$ or $\mathrm{Cr}$ ), conductive polymers as resists or discharge layers and ion shower methods [1-3]. However, all of these methods either require additional process steps with possible material compatibility problems or limit the application to specific types of resist systems.

The variable pressure scanning electron microscope (VPSEM) has been utilized for a number of applications in imaging and microanalysis of electrically insulating materials. The environmental gas in the VPSEM chamber is ionized upon electron impact and the effects of surface charge on secondary electron (SE) imaging are minimized through the neutralizing action of positive gas ions. A secondary implication of the environmental gas is the scattering of the primary electron beam leading to the formation of an electron "skirt" around the primary beam. Paul applied the same charge-balance concept in a recurrent-EBL process for the production of MEMS devices and studied the effects of gas pressure on pattern linewidths [4]. However, to our knowledge the technique of variable pressure $e \mathrm{BL}$ (VP-eBL) has not yet been applied to the fabrication of smaller structures $(<100 \mathrm{~nm})$.

We present the use of VP-eBL for the direct patterning of nanometer-scale structures on insulating substrates in the absence of a conductive coating. The distortion-free electrode pattern in Figure $1 \mathrm{~b}$ was fabricated using VP-eBL under the same conditions as that in Figure 1a, with the notable exception of a chamber pressure of 1 Torr. We have studied the effects of gas pressure $\left(\mathrm{H}_{2} \mathrm{O}\right)$, working distance (beam-gas path length), beam energy and detector bias on proximity dose, linewidth and exposure dose. One of the more intriguing results, shown in Figure 2, indicates that pattern linewidths seem to decrease with increasing gas pressure and working distance. This preliminary result contradicts the previous work by Paul (at least for the small beam currents required for these high resolution patterns) and is in conflict with conventional wisdom and intuition, which suggest that the beam skirting effect should increase linewidth. These results were used to develop a refined process capable of patterning electrodes with linewidths approaching those achieved in our system under high vacuum conditions on conductive substrates. The VPSEM images in Figure 3 show patterned $\mathrm{Au} / \mathrm{Pd}$ electrodes on glass substrates with $20 \mathrm{~nm}$ linewidths and $10 \mathrm{~nm}$ gaps. VP- $e \mathrm{BL}$ is a robust technique which allows for direct patterning on insulating substrates 
using conventional $e \mathrm{BL}$ resists and processing. As a result of this capability, VP-eBL may be useful for patterning on substrates such as sapphire, $\mathrm{MgO}$ and polymers with potential applications in optoelectronics and flexible electronics. In addition, further characterization of the VP- $e$ BL process may provide insight into the mechanisms electron scattering and charge-balance in the VPSEM.

References

[1] D. R. S. Cumming et al., J. Vac. Sci. Technol. B. 15 (1997) 2859.

[2] M. Angelopoulos et al., J. Vac. Sci. Technol. B. 11 (1993) 2794.

[3] T. Sakashita et al., J. Vac. Sci. Technol. B. 7 (1989) 1528.

[4] B. K. Paul, Scanning 19 (1997) 466.

[5] This research is partially supported by NUANCE staff development program and NU-NSEC.
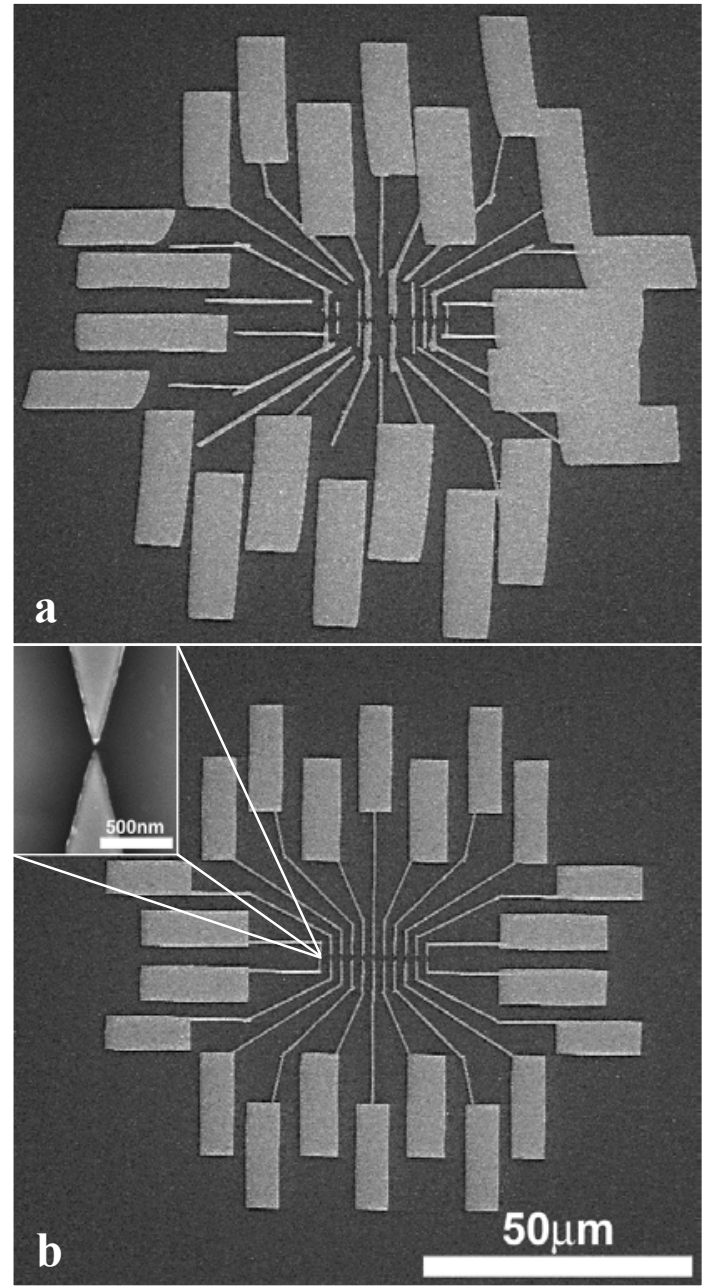

Figure 1. VPSEM images of $\mathrm{Au} / \mathrm{Pd}$ electrodes patterned via $e$ BL and lift-off processing in PMMA on glass with a $30 \mathrm{kV}$ beam in high vacuum (a) and with the VP-eBL using 1 Torr of water vapor (b). The inset in (b) is a close-up of the $\sim 30 \mathrm{~nm}$ electrode gap.

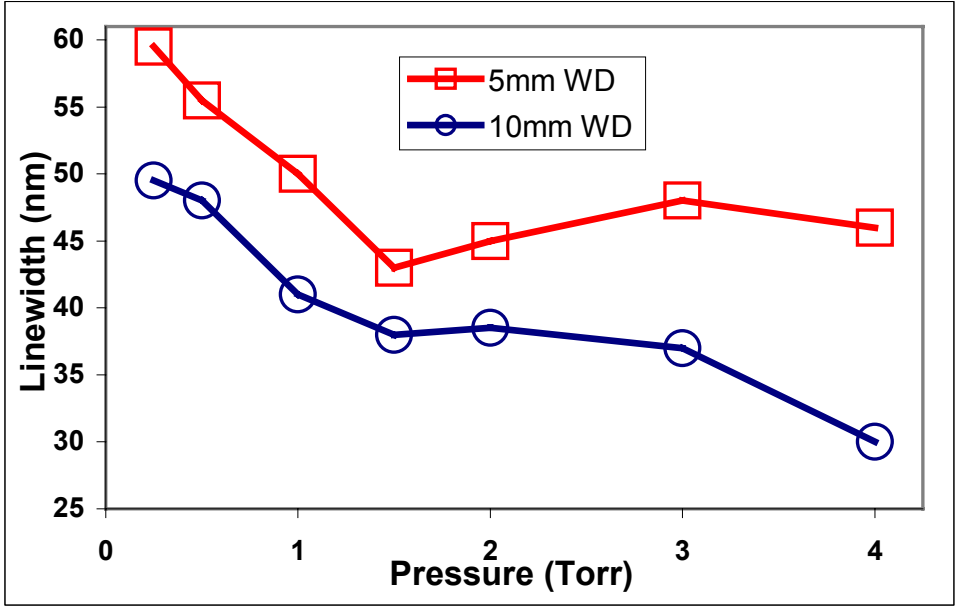

Figure 2. Variation in pattern linewidth with chamber pressure and working distance (WD) for $15 \mathrm{kV}$ VP- $e \mathrm{BL}$ process. (Note: beam-gas path length is less than WD due to the presence of a pressure-limiting aperture)

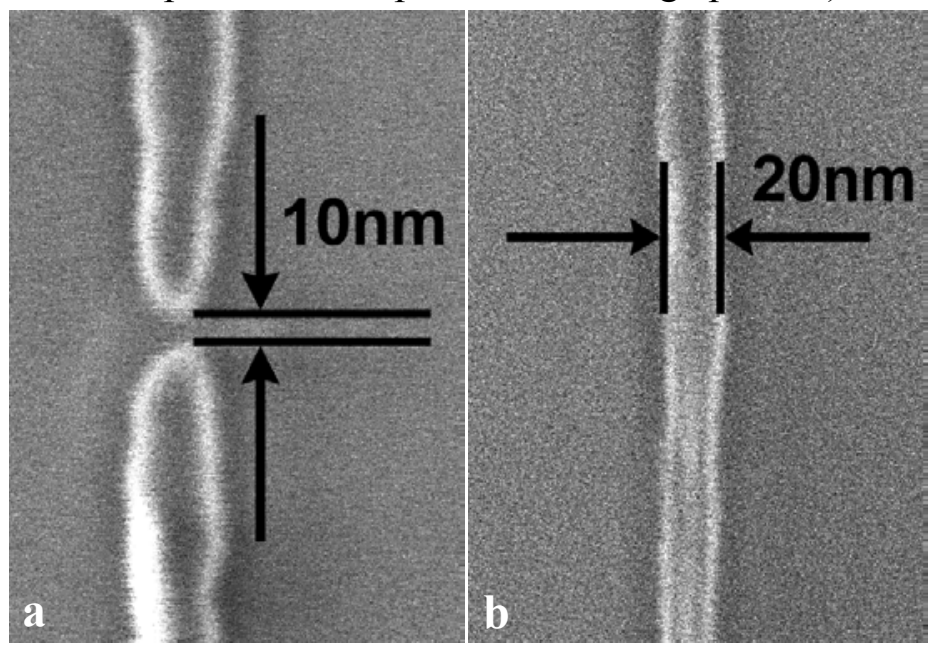

Figure 3. VPSEM images of small features patterned by VP-eBL showing $\sim 20 \mathrm{~nm}$ linewidths (a) and $\sim 10 \mathrm{~nm}$ electrode gaps (b). 